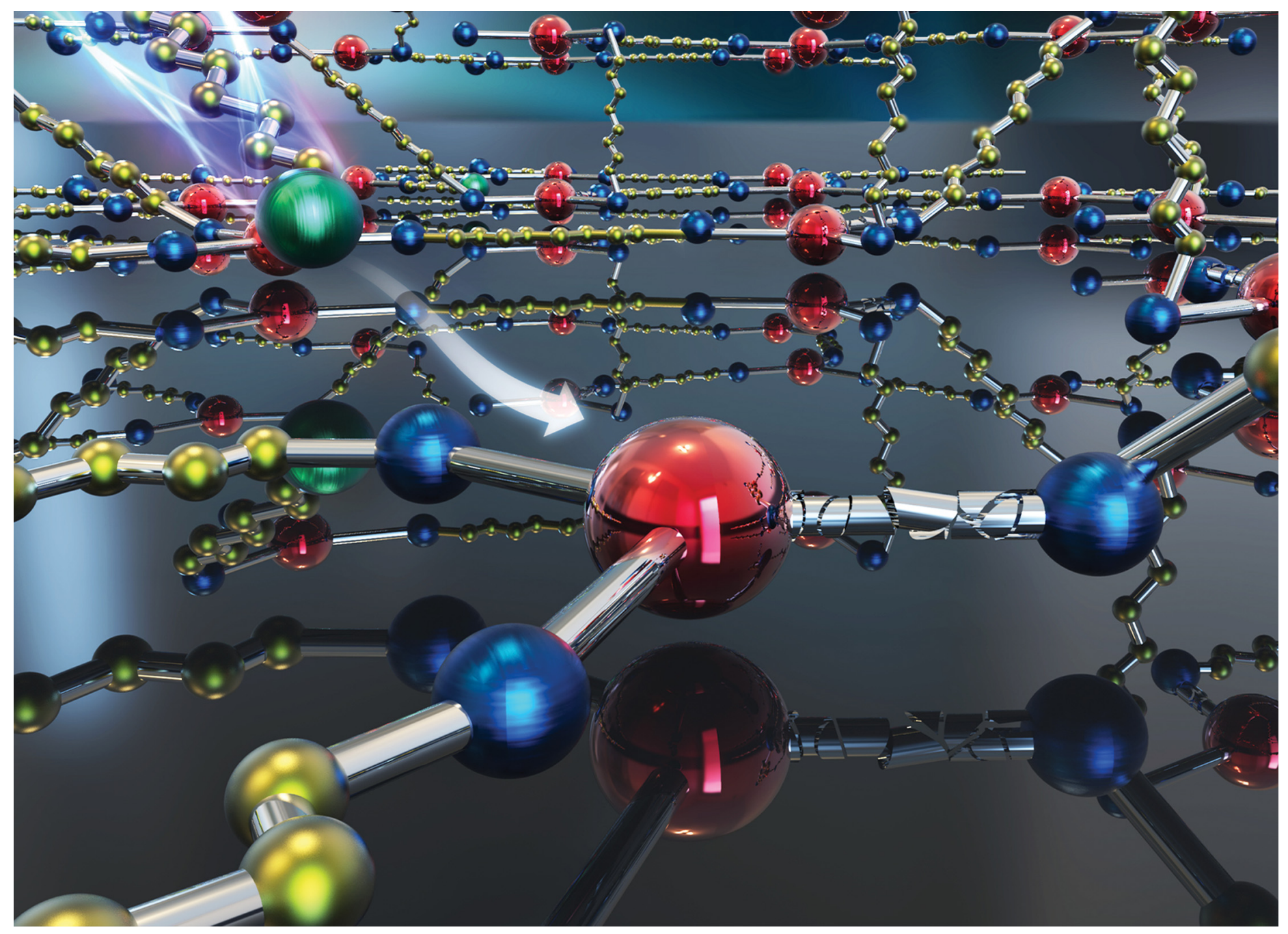

Highlighting research from the research group of Prof. Christopher Evans.

Dynamic polymer networks made from precise lengths of ethylene chains between boronic ester crosslinks

Effect of precise linker length, bond density, and broad temperature window on the rheological properties of ethylene vitrimers

Ethylene networks are investigated with precise spacings between dynamic covalent bonds. The activation energies for relaxation increase with crosslink density, and show deviations from Arrhenius behavior upon cooling towards the glass transition temperature.

\section{As featured in:}

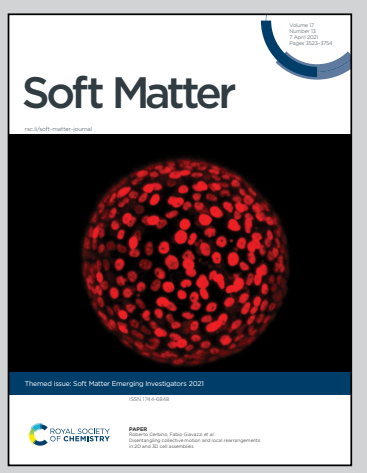

See Bhaskar Soman and

Christopher M. Evans,

Soft Matter, 2021, 17, 3569. 
Check for updates

Cite this: Soft Matter, 2021, 17, 3569

Received 26th August 2020,

Accepted 9th November 2020

DOI: $10.1039 / \mathrm{d} 0 \mathrm{sm01544j}$

rsc.li/soft-matter-journal

\title{
Effect of precise linker length, bond density, and broad temperature window on the rheological properties of ethylene vitrimers $\dagger$
}

\author{
Bhaskar Soman ${ }^{\mathrm{ab}}$ and Christopher M. Evans (D) *ab
}

\begin{abstract}
Dynamic networks which undergo topology conserving exchange reactions, sometimes called vitrimers, show properties intermediate to thermosets and thermoplastics. The dynamic nature of the networks results in complex rheological properties and has attracted much attention in the past decade for selfhealing, malleable and recyclable polymers. Here, we investigate a series of precise, high crosslink density telechelic ethylene vitrimers as a function of temperature and crosslink density. The networks show a rubbery plateau at high frequencies and a terminal flow regime at lower frequencies. With increasing crosslink density, the rubbery plateau modulus shows a monotonic increase and the terminal flow shifts to lower frequencies. The plateau modulus at high frequency increases as a function of temperature, as expected for a conserved network topology. When plotted against inverse temperature, the zero shear viscosities show a characteristic Arrhenius behavior, and the activation energy monotonically increases with crosslink density. Crossover frequency and shift factors (from time temperature superposition) also show Arrhenius behavior with activation energies in good agreement with those determined from zero shear viscosity. A positive deviation from this Arrhenius trend is observed beginning as high as $100 \mathrm{~K}$ above the glass transition temperature for $C_{6}$ and $C_{8}$ networks. Further investigations of such networks are critical for the development of sustainable and recyclable replacements for commercial plastics.
\end{abstract}

\section{Introduction}

Dynamic polymer networks including supramolecular polymers, ${ }^{1-9}$ covalent adaptable networks ${ }^{10-14}$ and vitrimers ${ }^{15-44}$ are being studied as self-healing, recyclable and sustainable polymers making them attractive materials to replace many commodity or functional plastics. Their unique properties are a consequence of the dynamic crosslinks which undergo reversible exchange reactions and allow for local rearrangements of network strands resulting in stress relaxation and flow. On short time scales the networks appear permanent and show a rubbery plateau, whereas on longer timescales they flow. The rheological properties of dynamic networks depend on the network architecture, crosslink density, crosslink chemistry, solvent, bond exchange kinetics and inherent segmental dynamics. While supramolecular polymers formed by secondary interactions such as hydrogen bonding, metal-ligand coordination

\footnotetext{
${ }^{a}$ Department of Materials Science and Engineering and Urbana, Illinois 61801, USA.E-mail: cme365@illinois.edu

${ }^{b}$ Frederick Seitz Materials Research Laboratory University of Illinois at Urbana-Champaign, Urbana, Illinois 61801, USA

$\dagger$ Electronic supplementary information (ESI) available. See DOI: 10.1039/ d0sm01544j
}

or host-guest interactions and have been studied for almost half a century, vitrimers differ in one key aspect where the crosslink junction is formed by dynamic covalent bonds which exchange in a topology conserving manner. The term vitrimer was coined by Leibler ${ }^{30}$ based on an Arrhenius temperature dependence of viscosity for polyester networks which is reminiscent of vitreous silica. It has been noted recently that this definition is not unique to dynamic networks with conserved exchange reactions and is also frequently observed in dissociative dynamic networks. ${ }^{45}$ Thus, there is still a need to understand under what conditions the mechanism of the exchange reaction will play a key role to the function or properties of dynamic networks. The rekindled interest in these networks in the last decade has led to numerous articles on vitrimers for shape memory polymers, ${ }^{35-37}$ shockwave dissipation, ${ }^{38}$ flame retardants, ${ }^{39}$ and solid electrolytes. ${ }^{40}$ While initial work was focused on stress relaxation times and self-healing as a function of bond chemistry and polymer backbone, recent work has moved to investigating the effects of crosslink density, ${ }^{41,42}$ defects $^{43}$ and solvent concentration ${ }^{44}$ on the rheological properties of vitrimers.

Two areas of vitrimer physics which have received little attention are the roles of extremely broad temperature windows and precise spacing between dynamic crosslinks. While the initial 
work of Leibler looked at a $180{ }^{\circ} \mathrm{C}$ temperature range, ${ }^{30}$ subsequent works have been largely limited to a less than $50{ }^{\circ} \mathrm{C}$ window. ${ }^{45}$ It is important to investigate wider windows because many polymer are processed well above their use temperatures, and it is unclear if the Arrhenius dependence will persist. Du Prez and coworkers found two distinct Arrhenius slopes in vinylogous urethane vitrimers ${ }^{17}$ attributed to a change in the dominant exchange mechanism with temperature. Additionally, many polymer are tested far above $T_{\mathrm{g}}$ where bond exchange is antipacted to control the macroscopic stress relaxation. It has been suggested ${ }^{15}$ that viscosity will show increasing deviations from Arrhenius behavior when approaching $T_{\mathrm{g}}$, but little experimental data exists in this regime.

The crosslink density in vitrimers has been investigated showing how the stress relaxation times and creep are impacted. $^{31-34}$ In supramolecular or dissociative networks, scaling relationships have been derived ${ }^{46,47}$ and measured experimentally ${ }^{48}$ but similar works do not yet exist for vitrimeric materials. Additionally, in prior works there is a distribution of molecular weights between crosslinks and the role of exact carbon spacers is unknown. Recent work from our group on permanent networks showed that odd-even effects can emerge in amorphous materials when the linkers are precise, and $T_{\mathrm{g}}$ jumps up and down as the carbon number systematically increases from 4 to $12 .{ }^{49}$ Winey and coworkers ${ }^{50}$ have also shown the importance of precision spacing of ionic groups along a polyethylene backone which can crystallize and form favorable structures for proton conduction. In the context of vitrimers, an understanding of how precise spacing of dynamic bonds impacts the macroscopic rheology is lacking.

Here, we report ethylene vitrimers synthesized by step growth polymerization of telechelic alkane diols and boric acid. A series of precise networks with varying crosslink densities were synthesized with exactly $6,8,10$ and 12 carbons $\left(\mathrm{C}_{6}-\mathrm{C}_{12}\right)$ between boronic ester crosslinking junctions. All networks have sub-ambient glass transition temperatures and are malleable at room temperature. Oscillatory shear rheology shows an increasing plateau modulus as a function of temperature, providing phenomenological evidence that the topology is conserved in the networks. Increasing crosslink density leads to a monotonic increase in the rubbery modulus and a delay of the terminal relaxation. The zero-shear viscosity, shift factors and crossover frequencies show characteristic Arrhenius behavior when measured far above $T_{\mathrm{g}}$. The activation energies $\left(E_{\mathrm{a}}\right)$ calculated from the fits are in good agreement with each other and systematically increase with crosslink density. The values are lower than reported for prior boronic ester vitrimers, ${ }^{51}$ which we attribute to the telechelic nature of the network. With decreasing temperature, the zero-shear viscosities show positive deviations from Arrhenius behavior as the glass transition is approached indicating an increasing role of segmental dynamics on the rheology. We also discuss the importance of broad temperature measurements in the context of determining topology freezing temperatures of dynamic networks.

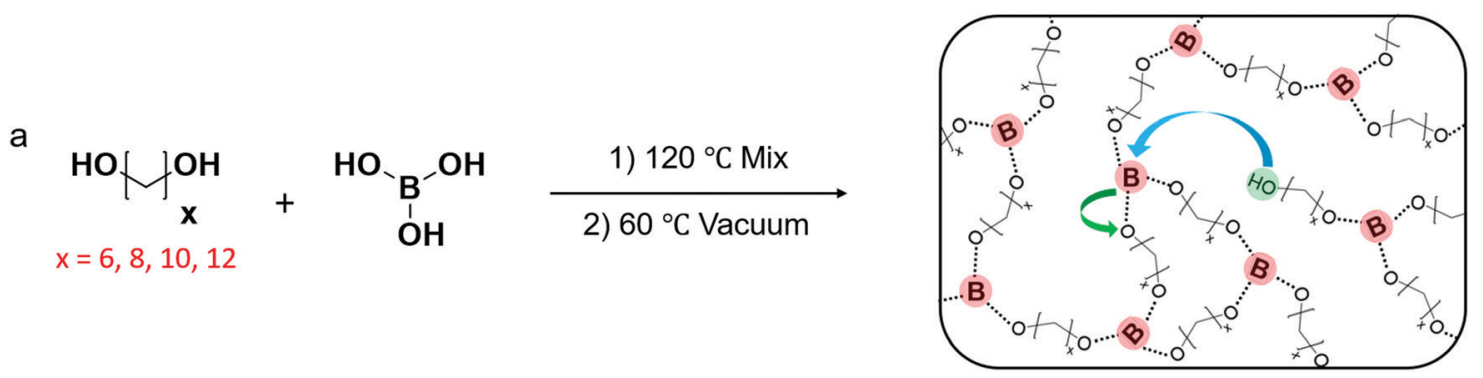

b

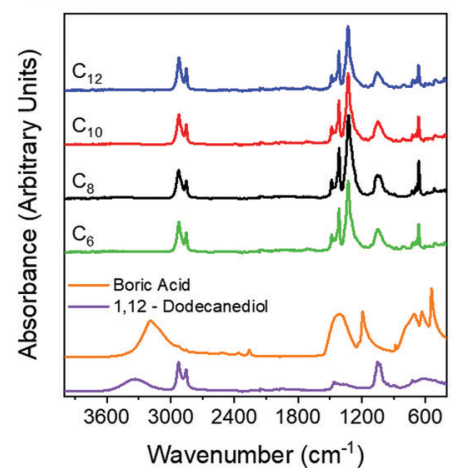

C

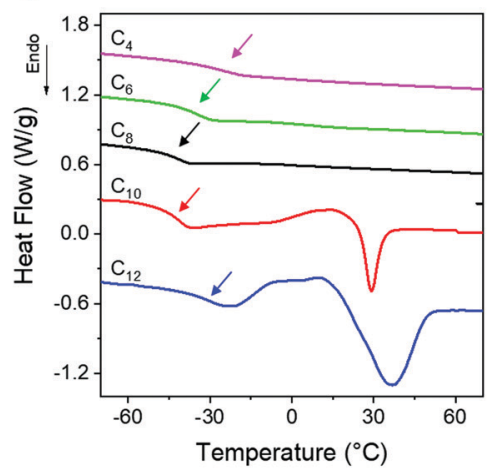

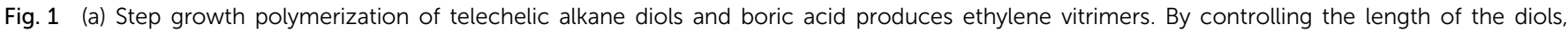

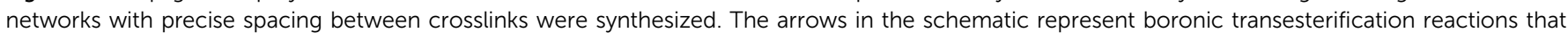

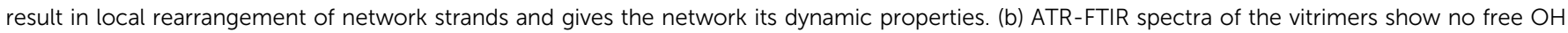

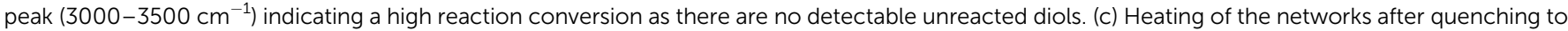

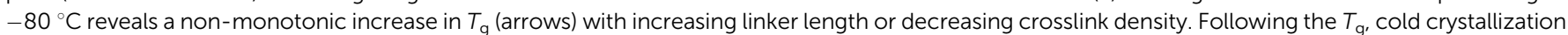
and melting transition were observed in $C_{10}$ and $C_{12}$ networks. 


\section{Results and discussion}

Dynamic PE networks were synthesized by the step growth polymerization of precise telechelic alkane diols and boric acid. Networks with exactly 6, 8, 10 and 12 carbons $\left(\mathrm{C}_{6}, \mathrm{C}_{8}, \mathrm{C}_{10}, \mathrm{C}_{12}\right)$ between boronic ester crosslinking junctions were prepared (Fig. 1a). Fourier transform infrared spectroscopy (FTIR) of the networks was performed at $80{ }^{\circ} \mathrm{C}$ to confirm the absence of the broad $\mathrm{OH}$ peak in the $3000 \mathrm{~cm}^{-1}$ to $3500 \mathrm{~cm}^{-1}$ range corresponding to unreacted species of the diols and boric acid. Within the sensitivity of the instrument, no $\mathrm{OH}$ peak is detected indicating that the network formation reached high conversion (Fig. 1b). The emergence of a sharp peak at $1300 \mathrm{~cm}^{-1}$ corresponds to the asymmetric $\mathrm{B}-\mathrm{O}$ stretch $^{52}$ indicating the formation of boronic ester crosslinks.

Thermal properties of the networks were investigated using differential scanning calorimetry (DSC) by first rapidly quenching from $150{ }^{\circ} \mathrm{C}$ to $-80{ }^{\circ} \mathrm{C}$ and then heating at $20{ }^{\circ} \mathrm{C} \min ^{-1}$. The $\mathrm{C}_{6}$ and $\mathrm{C}_{8}$ networks show no melting transitions while the $\mathrm{C}_{10}$ and $\mathrm{C}_{12}$ networks show cold crystallization followed by a melting transition with peak melting temperatures $\left(T_{\mathrm{m}}\right)$ in the
25-30 ${ }^{\circ} \mathrm{C}$ range. The glass transition temperatures $\left(T_{\mathrm{g}}\right)$ of the networks are $-27{ }^{\circ} \mathrm{C}\left(\mathrm{C}_{4}\right),-36{ }^{\circ} \mathrm{C}\left(\mathrm{C}_{6}\right),-43{ }^{\circ} \mathrm{C}\left(\mathrm{C}_{8}\right),-41{ }^{\circ} \mathrm{C}\left(\mathrm{C}_{10}\right)$ and $-32{ }^{\circ} \mathrm{C}\left(\mathrm{C}_{12}\right)$ (Fig. 1c). The increase in crosslink density is expected to lead to a monotonic increase in $T_{\mathrm{g}},{ }^{49}$ but a reproducible non-monotonic trend is observed. This is correlated with the appearance of crystallization during quenching and cold crystallization on heating in the $\mathrm{C}_{10}$ and $\mathrm{C}_{12}$ networks (Fig. S1, ESI †). No crystallization is observed in the heating or cooling cycles for $\mathrm{C}_{4}, \mathrm{C}_{6}$ and $\mathrm{C}_{8}$ networks. The crystalline domains may provide greater area for the rigid amorphous fraction to form which hinders segmental dynamics and raises $T_{\mathrm{g}}$. All subsequent measurements were performed above any melting transitions to avoid competing phenomena.

Oscillatory shear rheology was used to probe the storage $\left(G^{\prime}\right)$ and loss $\left(G^{\prime \prime}\right)$ modulus of the PE networks. Since the $\mathrm{C}_{10}$ and $\mathrm{C}_{12}$ networks are crystalline at room temperature, all experiments were performed above $40{ }^{\circ} \mathrm{C}$ to prevent the interference of crystallization on rheological measurements. Frequency sweeps were performed at $10{ }^{\circ} \mathrm{C}$ intervals from $40{ }^{\circ} \mathrm{C}$ to $140{ }^{\circ} \mathrm{C}$. The ethylene networks show a solid like $\left(G^{\prime}>G^{\prime \prime}\right)$ rubbery response at high frequencies and the plateau modulus
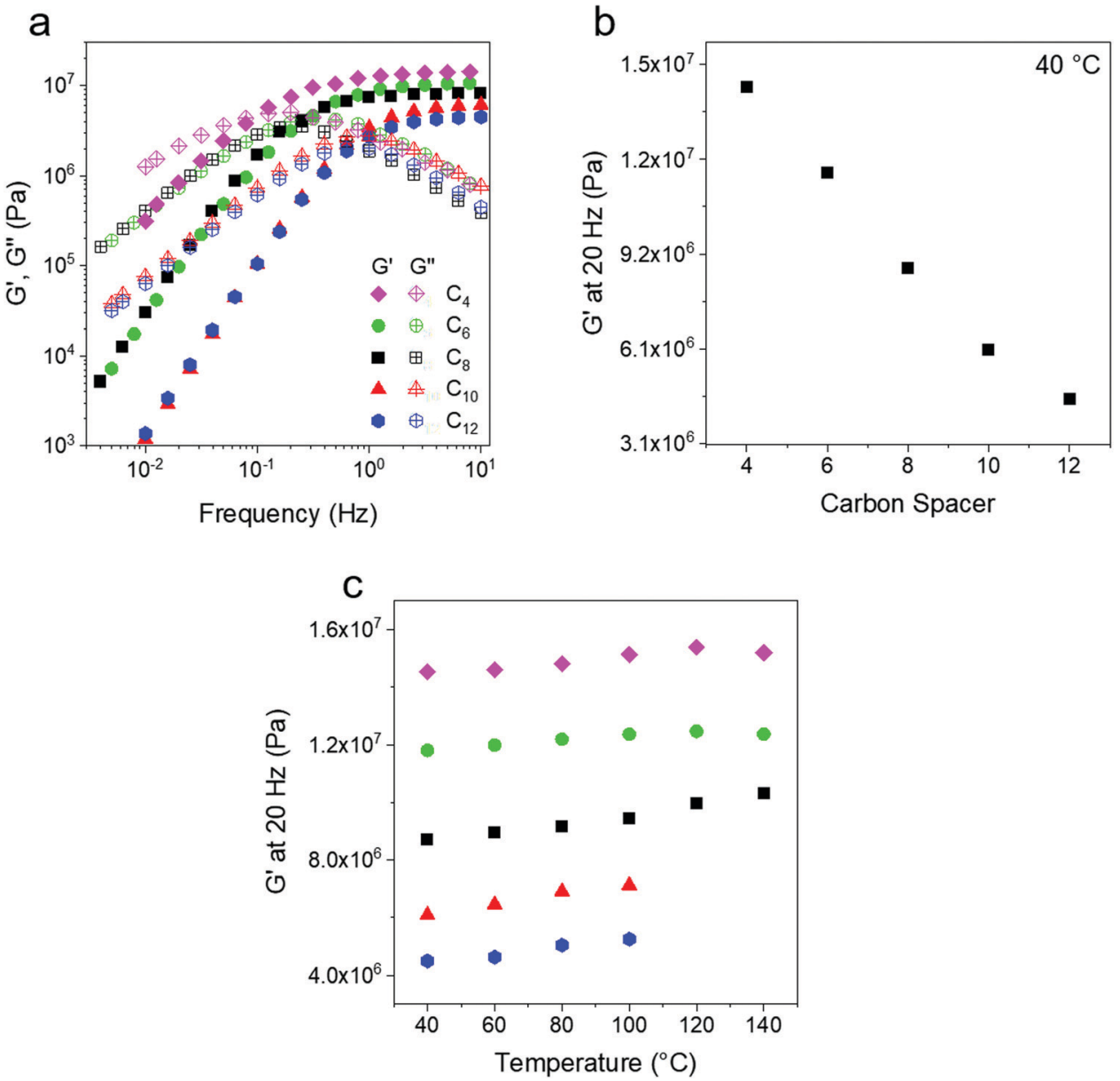

Fig. 2 (a) Storage and loss moduli of ethylene vitrimers at $40{ }^{\circ} \mathrm{C}$. A monotonic increase in rubbery plateau modulus at high frequency is observed with increasing crosslinking density. The terminal relaxation shifts to lower frequency as the crosslink density increases. (b) A comparison of the rubbery modulus with increasing linker length (decreasing crosslink density) shows a linear decrease as anticipated. (c) The rubbery modulus of the networks also shows a slight increase with increasing temperature as expected for networks with conserved topology. Symbols are the same as in (a). 
at $40{ }^{\circ} \mathrm{C}$ varies from $\sim 4$ to $14 \mathrm{MPa}$ depending on crosslink density (Fig. 2a). This is expected of a rubbery network ${ }^{53}$ and similar results have been shown in other experimental works on dynamic networks. ${ }^{54}$ Although some of the data overlap at low frequency, the modulus at $20 \mathrm{~Hz}$ changes proportionally to the carbon spacer as expected (Fig. 2b). At lower frequencies a terminal flow regime with characteristic slopes of $\sim 2$ for $G^{\prime}$ and $\sim 1$ for $G^{\prime \prime}$ (Fig. S2, ESI $\dagger$ ) is seen for all temperature. The terminal relaxation shifts to lower frequencies with increasing crosslink density, as has been previously reported for other telechelic systems. ${ }^{41,55}$ The modulus measured at a constant frequency of $20 \mathrm{~Hz}$ also increases with temperature, as expected for systems with conserved network topology due to entropic elasticity. ${ }^{52}$

Master curves of the storage and loss modulus (Fig. 3) are made by horizontally shifting individual frequency sweeps onto a reference curve $\left(T_{\text {ref }}=140{ }^{\circ} \mathrm{C}\right)$ using the time temperature superposition (TTS) principle. On a seven order of magnitude log scale, the plateau appears constant; however, a closer inspection reveals that the modulus does increase with temperature, particularly at higher frequency (Fig. 2c and Fig. S3, $\mathrm{ESI} \dagger)$. Boronic transesterification exchange reactions proceed in a topology conserving manner as shown by several groups ${ }^{51,56,57}$ and thus this increase is expected. In prior work, polyethylene oxide dynamic networks with the same boronic ester bonds as the present work also showed an increase in modulus on heating. ${ }^{40}$ This trend is in contrast to dissociative dynamic networks where modulus decreases with temperature. ${ }^{13,45}$

From the horizontal shifting of frequency sweeps, shift factors $\left(a_{T}\right)$ were obtained and plotted for all networks and show a systematic increase in the slope, and thus activation energy, from an Arrhenius fit (Fig. 4a). Next, the zero shear viscosity of the networks was calculated from 140 to $0{ }^{\circ} \mathrm{C}$ using the low frequency slope of the loss modulus, ${ }^{58}$ and a monotonic increase with increasing crosslink density is observed (Fig. 4b). Complex viscosity was also calculated and compared to this method, with both approaches yielding identical results within error (Fig. S4, ESI $\dagger$ ). Viscosity plotted against inverse temperature was fit to an Arrhenius expression, and shows quantitative agreement with the values from $a_{T}$ data (Table 1) indicating that they both reflect the same macroscopic flow controlled by dynamic bond exchange. A third metric, the crossover frequency ( $\omega_{\text {crossover }}$ ), was also examined and defined where $G^{\prime}=G^{\prime \prime}$ corresponding to the transition from a viscoelastic solid
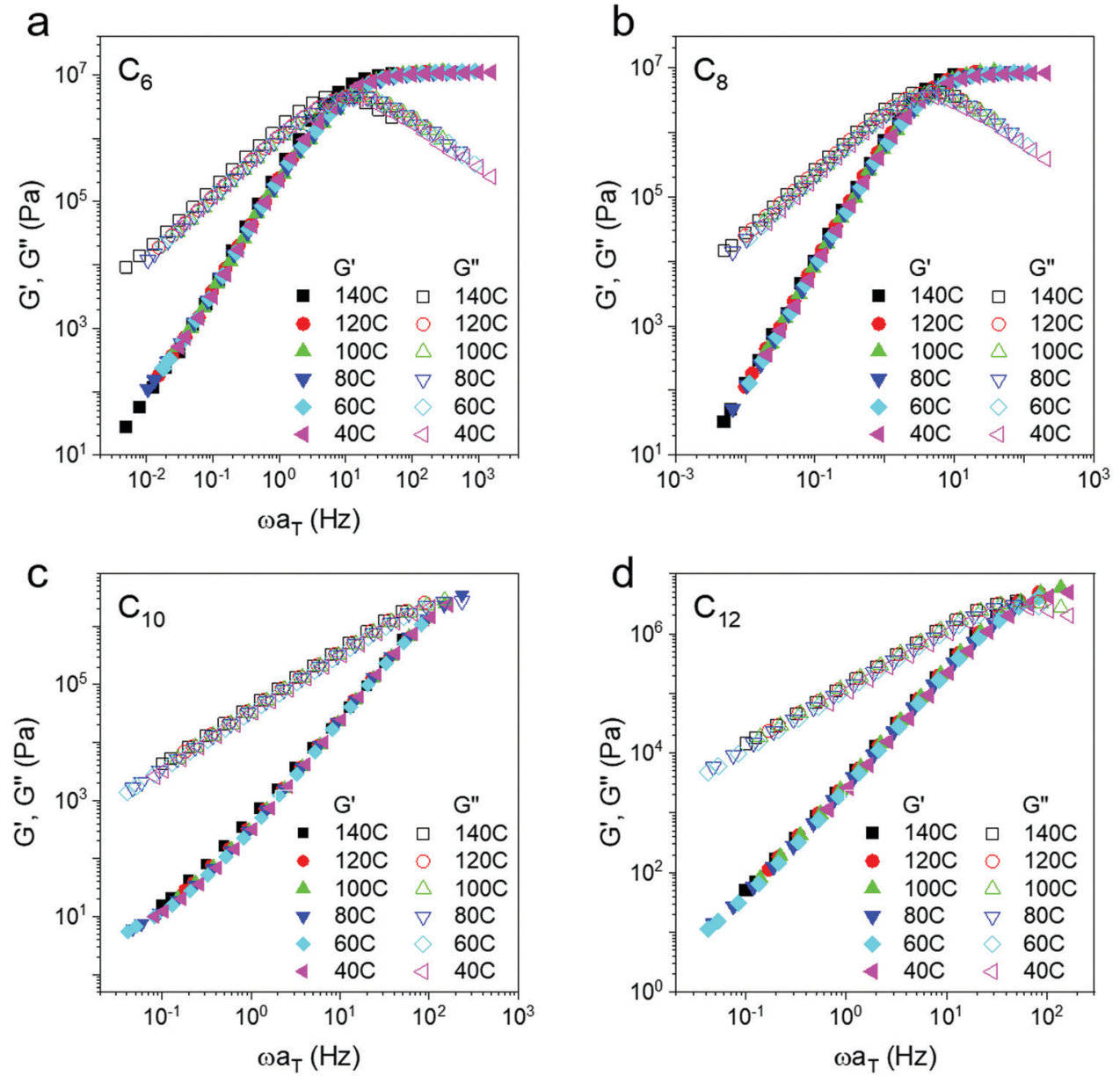

Fig. 3 Horizontally shifted master curves $\left(T_{\text {ref }}=140{ }^{\circ} \mathrm{C}\right)$ of the networks. The high frequency rubbery modulus remains constant with increasing temperature, intermediate to expectations for permanent and dissociative dynamic networks. 
a

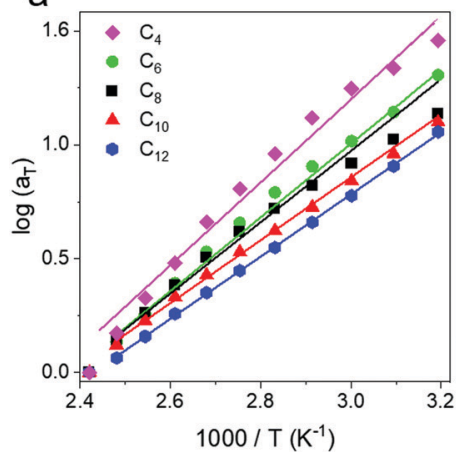

b

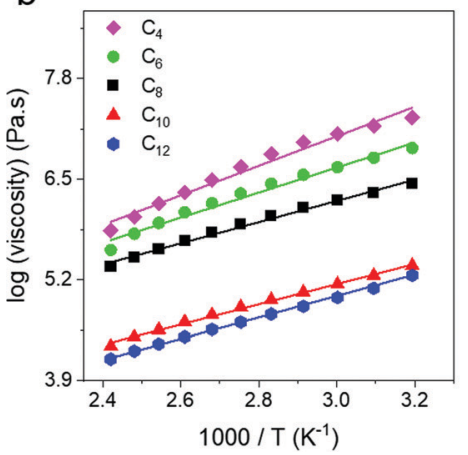

C

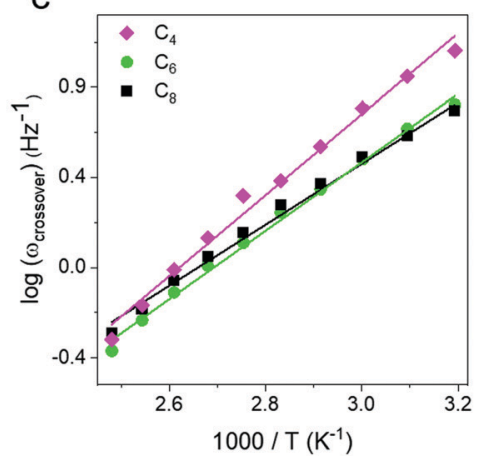

Fig. 4 (a) Shift factors, (b) zero shear viscosity and (c) crossover frequency as a function of inverse temperature. All quantities show an Arrhenius behavior in the $100{ }^{\circ} \mathrm{C}$ temperature window.

to a viscoelastic liquid. In the case of $\mathrm{C}_{10}$ and $\mathrm{C}_{12}$, a crossover frequency could not be measured at temperatures above $60{ }^{\circ} \mathrm{C}$ because even at the highest frequencies $G^{\prime \prime}>G^{\prime}$. For $\mathrm{C}_{4}, \mathrm{C}_{6}$ and $\mathrm{C}_{8}$ networks crossover frequencies were obtained as a function of temperature and are shown in Fig. 4c. Although $\omega_{\text {crossover was }}$ only obtained in three networks, it is also in good agreement with zero shear viscosity and shift factor in reflecting the activation energy associated with flow. In the case of vitrimers which obey time-temperature superposition, the choice of analysis metric does not appear to matter for determining activation energies. The $E_{\text {a }}$ values for our telechelic networks are all lower than what has been reported for boronic ester vitrimers with pendent dynamic bonds along the backbone by Guan and coworkers who measured $E_{\mathrm{a}}=52.7 \mathrm{~kJ} \mathrm{~mol}^{-1} .^{51}$ Sokolov and coworkers have noted that in associating polymers, telechelic structures lead to lower bond breaking energies ${ }^{59}$ which is consistent with our findings. Additionally, we observe a major increase in $E_{\mathrm{a}}$ with increased crosslink density suggesting that exchange processes are easier in more open networks (Table 1). From the shift factor data $E_{\mathrm{a}} \sim N^{-0.4}$, and it will be important to push to higher linker lengths to determine where the value becomes molecular weight independent. It is also unclear at present how linker polydispersity will affect these values.

In addition to $T_{\mathrm{g}}$ and $T_{\mathrm{m}}$, a hypothetical topology freezing temperature $\left(T_{\mathrm{v}}\right)$ has been suggested for vitrimers where the bond exchange occurs on slow enough timescales that the network is frozen and $\eta=10^{12} \mathrm{~Pa} \mathrm{~s},{ }^{60}$ the same criterion

Table 1 Activation Energy from Arrhenius fits to shift factors, zero shear viscosity and crossover frequency

\begin{tabular}{llll}
\hline & \multicolumn{2}{l}{ Activation Energy, $E_{\mathrm{a}}\left(\mathrm{kJ} \mathrm{mol}^{-1}\right)$} \\
\cline { 2 - 4 } & Shift factors & Zero shear viscosity & $\omega_{\text {crossover }}$ \\
\hline $\mathrm{C}_{4}$ & 43.0 & 41.6 & 38.5 \\
$\mathrm{C}_{6}$ & $32.3 \pm 0.12$ & $28.9 \pm 0.12$ & $33.0 \pm 0.13$ \\
$\mathrm{C}_{8}$ & $32.2 \pm 0.15$ & $25.4 \pm 0.08$ & $28.9 \pm 0.10$ \\
$\mathrm{C}_{10}$ & $27.6 \pm 0.06$ & $25.6 \pm 0.08$ & $-{ }^{a}$ \\
$\mathrm{C}_{12}$ & $27.3 \pm 0.02$ & $26.4 \pm 0.03$ &
\end{tabular}

${ }^{a}$ Not observable. invoked for the glass to rubber transition. The position of the $T_{\mathrm{v}}$ with respect to the $T_{\mathrm{g}}$ and $T_{\mathrm{m}}$ may provide insights regarding the relative importance of bond exchange or segmental dynamics in controlling stress relaxation in different temperature regimes. ${ }^{61,62}$ The $T_{\mathrm{v}}$ of our networks was obtained by extrapolating the zero shear viscosities to a value of $10^{12} \mathrm{~Pa} \mathrm{~S}$ (Fig. 5a). We note this massive extrapolation is not rigorous but was used to mimic protocols in the vitrimers literature. ${ }^{62,63} \mathrm{We}$ view $T_{\mathrm{v}}$ as a potentially ambiguous temperature as it shares the same criteria as the glass transition (viscosity $=10^{12} \mathrm{~Pa} \mathrm{~s}$ ). Additionally, depending on the modulus the $T_{\mathrm{v}}$ could correspond to timescales of relaxation spanning $10^{8}$ to $10^{3} \mathrm{~s}$ for networks ranging from ultrasoft $(10 \mathrm{kPa})$ to glassy $(1 \mathrm{GPa})$ through the Maxwell relation $\eta=G \tau$. A topology freezing transition based on a relaxation time would seem more informative than a temperature, but it is unclear what timescale is appropriate.

The $T_{\mathrm{v}}$ values were determined as $-116{ }^{\circ} \mathrm{C}(\mathrm{C} 6),-135^{\circ} \mathrm{C}(\mathrm{C} 8)$, $-149{ }^{\circ} \mathrm{C}(\mathrm{C} 10)$ and $-143{ }^{\circ} \mathrm{C}(\mathrm{C} 12)$ which are all well below $T_{\mathrm{g}}$. Thus, upon cooling the $T_{\mathrm{g}}$ is expected to intervene and lead to an increase in viscosity above the extrapolation of an Arrhenius fit. Such a deviation is observed at $\sim T_{\mathrm{g}}+100{ }^{\circ} \mathrm{C}$, and corresponds to a transition from a regime controlled by exchange reactions to a regime where segmental dynamics begin to contribute to an increased viscosity (Fig. $5 \mathrm{~b}$ ). This can be clearly seen in $\mathrm{C}_{6}$ and $\mathrm{C}_{8}$ as their zero shear viscosities show a positive deviation from the Arrhenius trend on a $1000 \mathrm{~T}^{-1}$ plot (Fig. 5c). These networks remain amorphous on cooling (Fig. S1, ESI $\dagger$ ), while $\mathrm{C}_{10}$ and $\mathrm{C}_{12}$ crystallize and thus viscosity measurements were not made below a temperature of $40{ }^{\circ} \mathrm{C}$. Our findings are in contrast to the initial work of Leibler who found a single Arrhenius dependence of zero-shear viscosity from $T_{\mathrm{g}}$ to $T_{\mathrm{g}}+180{ }^{\circ} \mathrm{C}$, presumably because their $T_{\mathrm{v}}$ was higher than $T_{\mathrm{g}}{ }^{30}$ In the present work, the extrapolation based on high temperature gives a different $T_{\mathrm{v}}$ than would be determined from the lower temperature data, and emphasizes the importance of measuring relaxation over a broad temperature window. Understanding where deviations begin depending on the combination of dynamic bond and polymer chemistry will be an important direction for future research. 
a
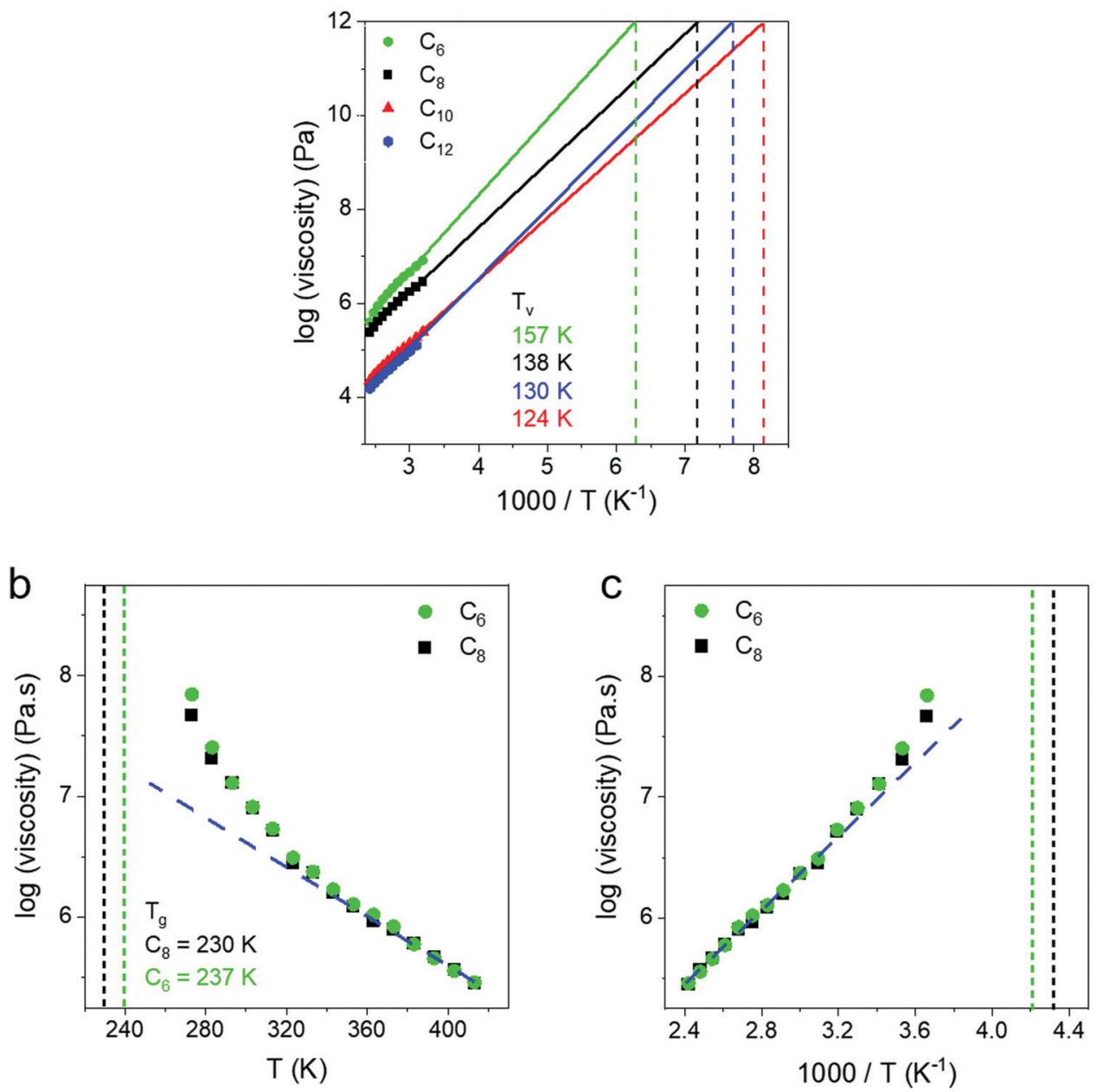

Fig. 5 (a) The topological freezing temperature $\left(T_{v}\right)$ is defined as the temperature at which the melt viscosity equals a value of $10^{12} \mathrm{~Pa} s$. We obtained the $T_{v}$ by extrapolating the Arrhenius zero shear viscosity fit to a value of $10^{12} \mathrm{~Pa} \mathrm{~s}$. (b) Zero shear viscosity vs. temperature and (c) inverse temperature. As the $T_{\mathrm{g}}$ is approached a positive deviation from the Arrhenius behavior is observed. The deviation indicates a transition from a regime controlled by exchange reactions to a regime where slow segmental dynamics begin to contribute to viscosity.

\section{Conclusion}

A series of precise telechelic ethylene vitrimers was investigated to understand the effect of temperature and precise crosslink density on their rheological properties. The dynamic nature of the networks manifests as a rubbery plateau at high frequencies and a low frequency terminal relaxation. Increasing crosslink density $\left(\mathrm{C}_{12}\right.$ to $\left.\mathrm{C}_{6}\right)$ leads to a monotonic increase in the rubbery modulus and a delay of the terminal relaxation. The zero shear viscosity shows a characteristic Arrhenius temperature dependence and monotonically increases with increasing crosslink density. Similar Arrhenius fits are made for the crossover frequency and shift factors, and the activation energies calculated from all metrics are in good agreement with each other. Telechelic networks show a lower activation energy than pendant networks with boronic esters. ${ }^{51}$ At high temperature, the rheological properties of the networks are dominated by the exchange reactions while a deviation from Arrhenius behavior is observed upon cooling. The additional increase in viscosity is attributed to the impending $T_{\mathrm{g}}$ and slowing segmental dynamics which begin to contribute as $T_{\mathrm{g}}+100 \mathrm{~K}$. These broad temperature window studies on model systems provide key insights to the understanding and application of vitrimers for a range of applications.

\section{Conflicts of interest}

There are no conflicts to declare.

\section{Acknowledgements}

Funding to support this work was provided by the Department of Materials Science and Engineering at the University of Illinois Urbana-Champaign. We also acknowledge the use of facilities at the Materials Research Laboratory. 


\section{References}

1 J.-M. Lehn, Supramolecular polymer chemistry-scope and perspectives, Polym. Int., 2002, 51(10), 825-839.

2 S. Seiffert and J. Sprakel, Physical chemistry of supramolecular polymer networks, Chem. Soc. Rev., 2012, 41(2), 909-930.

3 R. F. M. Lange, M. Van Gurp and E. W. Meijer, Hydrogenbonded supramolecular polymer networks, J. Polym. Sci., Part A: Polym. Chem., 1999, 37(19), 3657-3670.

4 X. Yan, D. Xu, X. Chi, J. Chen, S. Dong, X. Ding, Y. Yu and F. Huang, A Multiresponsive, Shape-Persistent, and Elastic Supramolecular Polymer Network Gel Constructed by Orthogonal Self-Assembly, Adv. Mater., 2012, 24(3), 362-369.

5 B. J. B. Folmer, R. P. Sijbesma, R. M. Versteegen, J. A. J. van der Rijt and E. W. Meijer, Supramolecular Polymer Materials: Chain Extension of Telechelic Polymers Using a Reactive Hydrogen-Bonding Synthon, Adv. Mater., 2000, 12(12), 874-878.

6 S. Dong, Y. Luo, X. Yan, B. Zheng, X. Ding, Y. Yu, Z. Ma, Q. Zhao and F. Huang, A Dual-Responsive Supramolecular Polymer Gel Formed by Crown Ether Based Molecular Recognition, Angew. Chem., Int. Ed., 2011, 50(8), 1905-1909.

7 S. Burattini, B. W. Greenland, D. H. Merino, W. Weng, J. Seppala, H. M. Colquhoun, W. Hayes, M. E. Mackay, I. W. Hamley and S. J. Rowan, A Healable Supramolecular Polymer Blend Based on Aromatic $\pi-\pi$ Stacking and Hydrogen-Bonding Interactions, J. Am. Chem. Soc., 2010, 132(34), 12051-12058.

8 X. Dai, Y. Zhang, L. Gao, T. Bai, W. Wang, Y. Cui and W. Liu, A Mechanically Strong, Highly Stable, Thermoplastic, and Self-Healable Supramolecular Polymer Hydrogel, $A d v$. Mater., 2015, 27(23), 3566-3571.

9 Q. Zhang, Y.-X. Deng, H.-X. Luo, C.-Y. Shi, G. M. Geise, B. L. Feringa, H. Tian and D.-H. Qu, Assembling a Natural Small Molecule into a Supramolecular Network with High Structural Order and Dynamic Functions, J. Am. Chem. Soc., 2019, 141(32), 12804-12814.

10 M. Delahaye, J. M. Winne and F. E. Du Prez, Internal Catalysis in Covalent Adaptable Networks: Phthalate Monoester Transesterification As a Versatile Dynamic Cross-Linking Chemistry, J. Am. Chem. Soc., 2019, 141(38), 15277-15287.

11 B. M. Richardson, D. G. Wilcox, M. A. Randolph and K. S. Anseth, Hydrazone covalent adaptable networks modulate extracellular matrix deposition for cartilage tissue engineering, Acta Biomater., 2019, 83, 71-82.

12 N. Van Herck, D. Maes, K. Unal, M. Guerre, J. M. Winne and F. E. Du Prez, Covalent Adaptable Networks with Tunable Exchange Rates Based on Reversible Thiol-yne CrossLinking, Angew. Chem., Int. Ed., 2020, 59(9), 3609-3617.

13 C. J. Kloxin and C. N. Bowman, Covalent adaptable networks: smart, reconfigurable and responsive network systems, Chem. Soc. Rev., 2013, 42(17), 7161-7173.

14 K. M. Lindenmeyer, R. D. Johnson and K. M. Miller, Selfhealing behaviour of furan-maleimide poly(ionic liquid) covalent adaptable networks, Polym. Chem., 2020, 11, 5321-5326.
15 W. Denissen, J. M. Winne and F. E. Du Prez, Vitrimers: permanent organic networks with glass-like fluidity, Chem. Sci., 2016, 7(1), 30-38.

16 A. Gablier, M. O. Saed and E. M. Terentjev, Rates of transesterification in epoxy-thiol vitrimers, Soft Matter, 2020, 16(22), 5195-5202.

17 M. Guerre, C. Taplan, R. Nicolaÿ, J. M. Winne and F. E. Du Prez, Fluorinated Vitrimer Elastomers with a Dual Temperature Response, J. Am. Chem. Soc., 2018, 140(41), 13272-13284.

18 A. Jourdain, R. Asbai, O. Anaya, M. M. Chehimi, E. Drockenmuller and D. Montarnal, Rheological Properties of Covalent Adaptable Networks with 1,2,3-Triazolium Cross-Links: The Missing Link between Vitrimers and Dissociative Networks, Macromolecules, 2020, 53, 1884-1900.

19 S. Kaiser, S. Wurzer, G. Pilz, W. Kern and S. Schlögl, Stress relaxation and thermally adaptable properties in vitrimerlike elastomers from HXNBR rubber with covalent bonds, Soft Matter, 2019, 15(30), 6062-6072.

20 R. Long, H. J. Qi and M. L. Dunn, Modeling the mechanics of covalently adaptable polymer networks with temperaturedependent bond exchange reactions, Soft Matter, 2013, 9(15), 4083-4096.

21 M. M. Obadia, A. Jourdain, P. Cassagnau, D. Montarnal and E. Drockenmuller, Tuning the Viscosity Profile of Ionic Vitrimers Incorporating 1,2,3-Triazolium Cross-Links, Adv. Funct. Mater., 2017, 27(45), 1-10.

22 R. G. Ricarte, F. Tournilhac, M. Cloître and L. Leibler, Linear Viscoelasticity and Flow of Self-Assembled Vitrimers: The Case of a Polyethylene/Dioxaborolane System, Macromolecules, 2020, 53(5), 1852-1866.

23 F. Snijkers, R. Pasquino and A. Maffezzoli, Curing and viscoelasticity of vitrimers, Soft Matter, 2017, 13(1), 258-268.

24 C. Taplan, M. Guerre, J. M. Winne and F. E. Du Prez, Fast processing of highly crosslinked, low-viscosity vitrimers, Mater. Horiz., 2020, 7(1), 104-110.

25 Y. Zhou, J. G. P. Goossens, R. P. Sijbesma and J. P. A. Heuts, Poly(butylene terephthalate)/Glycerol-based Vitrimers via Solid-State Polymerization, Macromolecules, 2017, 50(17), 6742-6751.

26 Y. Zhou, R. Groote, J. G. P. Goossens, R. P. Sijbesma and J. P. A. Heuts, Tuning PBT vitrimer properties by controlling the dynamics of the adaptable network, Polym. Chem., 2019, 10(1), 136-144.

27 H. Li, B. Zhang, K. Yu, C. Yuan, C. Zhou, M. L. Dunn, H. J. Qi, Q. Shi, Q.-H. Wei and J. Liu, Influence of treating parameters on thermomechanical properties of recycled epoxy-acid vitrimers, Soft Matter, 2020, 16(6), 1668-1677.

28 C. He, P. R. Christensen, T. J. Seguin, E. A. Dailing, B. M. Wood, R. K. Walde, K. A. Persson, T. P. Russell and B. A. Helms, Conformational Entropy as a Means to Control the Behavior of Poly(diketoenamine) Vitrimers In and Out of Equilibrium, Angew. Chem., Int. Ed., 2020, 59(2), 735-739.

29 M. Röttger, T. Domenech, R. van der Weegen, A. Breuillac, R. Nicolaÿ and L. Leibler, High-performance vitrimers from commodity thermoplastics through dioxaborolane metathesis, Science, 2017, 356(6333), 62. 
30 D. Montarnal, M. Capelot, F. Tournilhac and L. Leibler, Silica-Like Malleable Materials from Permanent Organic Networks, Science, 2011, 334(6058), 965.

31 T. Stukenbroeker, W. Wang, J. M. Winne, F. E. Du Prez, R. Nicolaÿ and L. Leibler, Polydimethylsiloxane quenchable vitrimers, Polym. Chem., 2017, 8(43), 6590-6593.

32 R. L. Snyder, D. J. Fortman, G. X. De Hoe, M. A. Hillmyer and W. R. Dichtel, Reprocessable Acid-Degradable Polycarbonate Vitrimers, Macromolecules, 2018, 51(2), 389-397.

33 M. Hayashi and R. Yano, Fair Investigation of Cross-Link Density Effects on the Bond-Exchange Properties for TransEsterification-Based Vitrimers with Identical Concentrations of Reactive Groups, Macromolecules, 2020, 53(1), 182-189.

34 L. Li, X. Chen, K. Jin and J. M. Torkelson, Vitrimers Designed Both To Strongly Suppress Creep and To Recover Original Cross-Link Density after Reprocessing: Quantitative Theory and Experiments, Macromolecules, 2018, 51(15), 5537-5546.

35 P. Yan, W. Zhao, B. Zhang, L. Jiang, S. Petcher, J. A. Smith, D. J. Parker, A. I. Cooper, J. Lei and T. Hasell, Inverse vulcanized polymers with shape memory, enhanced mechanical properties, and vitrimer behavior, Angew. Chem., Int. Ed., 2020, 59, 13371-13378.

36 F. Ji, X. Liu, D. Sheng and Y. Yang, Epoxy-vitrimer composites based on exchangeable aromatic disulfide bonds: reprocessibility, adhesive, multi-shape memory effect, Polymer, 2020, 122514.

37 X. Yang, L. Guo, X. Xu, S. Shang and H. Liu, A fully bio-based epoxy vitrimer: self-healing, triple-shape memory and reprocessing triggered by dynamic covalent bond exchange, Mater. Des., 2020, 186, 108248.

38 J. Lee, B. B. Jing, L. E. Porath, N. R. Sottos and C. M. Evans, Shock Wave Energy Dissipation in Catalyst-Free Poly (dimethylsiloxane) Vitrimers, Macromolecules, 2020, 53, 4741-4747.

39 L. Zhou, G. Zhang, Y. Feng, H. Zhang, J. Li and X. Shi, Design of a self-healing and flame-retardant cyclotriphosphazenebased epoxy vitrimer, J. Mater. Sci., 2018, 53(9), 7030-7047.

40 B. B. Jing and C. M. Evans, Catalyst-Free Dynamic Networks for Recyclable, Self-Healing Solid Polymer Electrolytes, J. Am. Chem. Soc., 2019, 141(48), 18932-18937.

41 Y. Liu, Z. Tang, J. Chen, J. Xiong, D. Wang, S. Wang, S. Wu and B. Guo, Tuning the mechanical and dynamic properties of imine bond crosslinked elastomeric vitrimers by manipulating the crosslinking degree, Polym. Chem., 2020, 11(7), 1348-1355.

42 R. Hajj, A. Duval, S. Dhers and L. Avérous, Network Design to Control Polyimine Vitrimer Properties: Physical Versus Chemical Approach, Macromolecules, 2020, 53, 3796-3805.

43 S. Ciarella, F. Sciortino and W. G. Ellenbroek, Dynamics of Vitrimers: Defects as a Highway to Stress Relaxation, Phys. Rev. Lett., 2018, 121(5), 58003.

44 M. Vatankhah-Varnoosfaderani, S. Hashmi, F. J. Stadler and A. GhavamiNejad, Mussel-inspired 3D networks with stiffirreversible or soft-reversible characteristics - It's all a matter of solvent, Polym. Test., 2017, 62, 96-101.
45 B. R. Elling and W. R. Dichtel, Reprocessable cross-linked polymer networks: are associative exchange mechanisms desirable?, ACS Cent. Sci., 2020, 6, 1488-1496.

46 G. Marrucci, S. Bhargava and S. L. Cooper, Models of shearthickening behavior in physically crosslinked networks, Macromolecules, 1993, 26(24), 6483-6488.

47 L. Leibler, M. Rubinstein and R. H. Colby, Dynamics of reversible networks, Macromolecules, 1991, 24(16), 4701-4707.

48 D. Xu and S. L. Craig, Scaling Laws in Supramolecular Polymer Networks, Macromolecules, 2011, 44(13), 5465-5472.

49 C. Shen, Q. Zhao and C. M. Evans, Ion specific, odd-even glass transition temperatures and conductivities in precise network polymerized ionic liquids, Mol. Syst. Des. Eng., 2019, 4(2), 332-341.

50 E. B. Trigg, T. W. Gaines, M. Maréchal, D. E. Moed, P. Rannou, K. B. Wagener, M. J. Stevens and K. I. Winey, Self-assembled highly ordered acid layers in precisely sulfonated polyethylene produce efficient proton transport, Nat. Mater., 2018, 17(8), 725-731.

51 O. R. Cromwell, J. Chung and Z. Guan, Malleable and SelfHealing Covalent Polymer Networks through Tunable Dynamic Boronic Ester Bonds, J. Am. Chem. Soc., 2015, 137(20), 6492-6495.

52 M. K. Smith and B. H. Northrop, Vibrational properties of boroxine anhydride and boronate ester materials: model systems for the diagnostic characterization of covalent organic frameworks, Chem. Mater., 2014, 26(12), 3781-3795.

53 H. M. James and E. Guth, Theory of the elastic properties of rubber, J. Chem. Phys., 1943, 11(10), 455-481.

54 T. Yan, K. Schröter, F. Herbst, W. H. Binder and T. ThurnAlbrecht, What Controls the Structure and the Linear and Nonlinear Rheological Properties of Dense, Dynamic Supramolecular Polymer Networks?, Macromolecules, 2017, 50(7), 2973-2985.

55 A. Breuillac, A. Kassalias and R. Nicolaÿ, Polybutadiene Vitrimers Based on Dioxaborolane Chemistry and Dual Networks with Static and Dynamic Cross-links, Macromolecules, 2019, 52(18), 7102-7113.

56 G. Springsteen and B. Wang, A detailed examination of boronic acid-diol complexation, Tetrahedron, 2002, 58(26), 5291-5300.

57 J. J. Cash, T. Kubo, A. P. Bapat and B. S. Sumerlin, RoomTemperature Self-Healing Polymers Based on DynamicCovalent Boronic Esters, Macromolecules, 2015, 48(7), 2098-2106.

58 R. L. Thompson, A. A. Alicke and P. R. de Souza Mendes, Model-based material functions for SAOS and LAOS analyses, J. Non-Newtonian Fluid Mech., 2015, 215, 19-30.

59 K. Xing, M. Tress, P.-F. Cao, F. Fan, S. Cheng, T. Saito and A. P. Sokolov, The Role of Chain-End Association Lifetime in Segmental and Chain Dynamics of Telechelic Polymers, Macromolecules, 2018, 51(21), 8561-8573.

60 H. Fang, W. Ye, Y. Ding and H. H. Winter, Rheology of the Critical Transition State of an Epoxy Vitrimer, Macromolecules, 2020, 53, 4855-4862. 
61 N. J. Van Zee and R. Nicolaÿ, Vitrimers: permanently crosslinked polymers with dynamic network topology, Prog. Polym. Sci., 2020, 104, 101233.

62 F. Gamardella, F. Guerrero, S. De la Flor, X. Ramis and A. Serra, A new class of vitrimers based on aliphatic poly(thiourethane) networks with shape memory and permanent shape reconfiguration, Eur. Polym. J., 2020, 122, 109361.

63 G. R. Lopez, L. Granado, G. l. Coquil, A. S. Lárez-Sosa, N. Louvain and B. Améduri, Perfluoropolyether (PFPE)based vitrimers with ionic conductivity, Macromolecules, 2019, 52(5), 2148-2155. 